\title{
Non-uniform Distributions of Quantum Particles in Multi-swarm Optimization for Dynamic Tasks
}

\author{
Krzysztof Trojanowski \\ Institute of Computer Science, Polish Academy of Sciences \\ Ordona 21, 01-237 Warsaw, Poland \\ trojanow@ipipan.waw.pl
}

\begin{abstract}
This paper presents research considering mixed multi-swarm optimization approach applied to dynamic environments. One of the versions of this approach, called mQSO is a subject of our special interest. The mQSO algorithm works with a set of particles divided into subswarms where every sub-swarm consists of two types of particles: classic and quantum ones. The research is focused on studying properties of the latter type. Two new distributions of new locations for the quantum particles are proposed: static and adaptive one. Both of them are based on an $\alpha$-stable symmetric distribution. In opposite to already published methods of distribution of new locations the proposed methods allow the locations to be distributed over the entire search space. Obtained results show high efficiency of the mQSO approach equipped with the proposed two new methods.
\end{abstract}

\section{Introduction}

In the presented research a mixed multi-swarm optimization in dynamic environments is studied. Application of mixed multi-swarm approach to dynamic optimization has already been tested and proved its efficiency. Approaches with static and varying number of subs-warms [1, 2, 3] as well as approaches with adaptive number of species in the swarm [4], [5], 6] have been researched. A version with static number of subs-warms called mQSO, where two types of particles: quantum particles and classic ones are in use and especially their rules of movement became a subject of our interest. Classic particles use velocity vectors to evaluate their new positions. The rules for quantum particles are based on the random distribution of possible new locations of a particle around its current location similarly to the distribution of the locations in the quantum cloud of the atom. The rules for quantum particles proposed in [2, 3] are based on the idea of uniform distribution over the space of a quantum cloud, which is a hyper-sphere of a constant radius with the current location in the middle.

In this paper we examine strategies of the quantum particle movement being alternative to the strategy mentioned above. Two methods of evaluation of new locations of the quantum particle are proposed and the efficiency of the mQSO equipped with these methods is experimentally verified. 
As a dynamic test-bed a MPB [7] generator was selected. In MPB we optimize in a real-valued 5-dimensional search space and the fitness landscape is built of a set of unimodal functions individually controlled by the parameters allowing to create different types of changes.

The paper is organized as follows. In Sec. 2 there is a brief presentation of the optimization algorithm. Two new methods of generation of the quantum particle's new locations are described in Sec. 3. Section 5 shows a measure used for evaluation of the results of experiments and the selected testing environment while Sec. [6 - the results of experiments performed with the environment. Section 7 concludes the presented research.

\section{Quantum Multi-swarm}

A simple scheme of the particle swarm optimization algorithm is given in Fig. 1. A PSO optimizer is equipped with a set of particles $\mathbf{x}_{i}$ where $i \in[1, \ldots N]$. Each of the particles represents a solution in an $n$-dimensional real valued search space. For the search space a fitness function $f(\cdot)$ is defined which is used to evaluate the quality of the solutions. A particle $\mathbf{y}_{i}$ represents the best solution found by the $i$-th particle (called particle attractor), and a particle $\mathbf{y}^{*}$ - the best solution found by the swarm (called swarm attractor). The scheme is made for maximization problem.

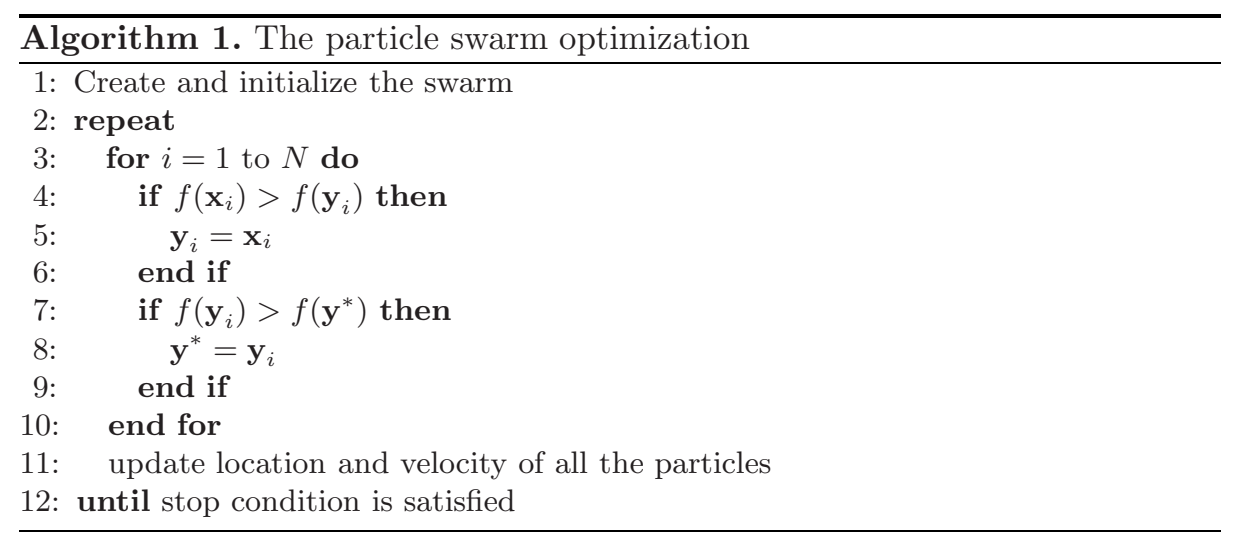

Search properties of the PSO scheme in Algorithm 1 are represented by the step "update location and velocity". In this step there are two main actions performed: first the velocity of each of the particles is updated and then all the particles change their location in the search space according to the new values of velocity vectors and the kinematic laws. Formally for every iteration $t$ of the search process every $j$-th coordinate of the vector of velocity $\mathbf{v}$ as well as the coordinate of the location $\mathbf{x}$ undergo the following transformation $[8]$ :

$$
\begin{aligned}
& v_{j}^{t+1}=\chi\left(v_{j}^{t}+c_{1} r_{1}^{t}\left(y_{j}^{t}-x_{j}^{t}\right)+c_{2} r_{2}^{t}\left(y_{j}^{* t}-x_{j}^{t}\right)\right), \\
& x_{j}^{t+1}=x_{j}^{t}+v_{j}^{t+1},
\end{aligned}
$$


where $r_{1}^{t}$ and $r_{2}^{t}$ are random values uniformly generated in the range [0,1], $\chi$ is a constriction factor and $\chi<1$ and $c_{1}$ and $c_{2}$ control the attraction to the best found personal and global solutions, respectively.

The basic idea presented in Algorithm 1 has been developed for non-stationary optimization applications. One of the first significant changes in this scheme is an introduction of multi-swarm. In the presented approach the number of subswarms is constant during the process of searching. Each of them is treated as an independent self-governing population which is not influenced by any of the neighbors. However, there are mechanisms which periodically perform some actions based on the information about the state of search of the entire swarm [3].

To guarantee the appropriate distribution of the sub-swarms over the entire search space the exclusion mechanism eliminates sub-swarms, which are located too close to each other. When the sub-swarms are too close to each other, the occupation of the same optimum is most likely to occur. In this case one of them is selected to be eliminated and a new one is generated from scratch. Any two subswarms are considered as located too close to each other if for the best solutions from the compared two sub-swarms the euclidean distance is closer than the defined threshold $\rho$. In 3 yet another mechanism of sub-swarms' management was proposed called anti-convergence, which protects against convergence of subswarms. However it was off in the presented experiments.

The last of the sub-swarms' management mechanisms described in [3] is based on mixing of types of particles in sub-swarms. In the presented research the mixed sub-swarms consist of two types of particles governed by two different rules of movement. While the location of the particles of the first type is evaluated according to classic formulas as discussed above, the remaining ones are treated as quantum particles and change their location according to the analogy with quantum dynamics of the particles. All the particles in such a mixed sub-swarm share the information about the current best position and the best position ever found by the sub-swarm.

Idea of quantum particle proposed by Blackwell and Branke in [2] originates from the quantum model of atom where the trajectories of electrons are described as quantum clouds. Adaptation of this idea to the model of movement of the particles rejects kinematics laws used in classic PSO for evaluation of a distance traveled by the particle with a constant velocity in a period of time. Instead of this a new position of the quantum particle is randomly generated inside a cloud of the given range $r_{\text {cloud }}$ surrounding $\mathbf{y}^{*}$ i.e. the current sub-swarm attractor. In quantum model the particle's speed becomes irrelevant, because every location inside the cloud can be chosen as a new location with a non-zero probability.

The model of quantum particles has been extended in this paper. Since the model proposed in 2] assumes the uniform distribution of the set of possible new locations of the particle over the cloud's space, it was interesting to test and verify another types of distributions. 


\section{Movement of Quantum Particles}

Two new types of distribution of new locations are considered in this paper. The first one is defined with static rules while the second one - adaptive rules. Both of them are based on two-phase mechanism. In the first phase a direction $\theta$ is selected. In the second phase a distance $d$ from the original is calculated.

The direction $\theta$ can be obtained with use of a random variable from the angularly uniform distribution on the surface of a hyper-sphere [9]. The distance $d$ is an $\alpha$-stable random variate and is computed as follows:

$$
\begin{aligned}
& d=S \alpha S(0, \sigma), \text { and } \\
& \sigma=r_{S \alpha S} \cdot\left(D_{w} / 2\right),
\end{aligned}
$$

where $S \alpha S(\cdot, \cdot)$ represents $\alpha$-stable symmetric distribution variate and $D_{w}$ is a width of the feasible part of the domain, i.e. a distance between a lower and an upper boundary of the search space. The new location is based on the found direction $\theta$ and a distance $d$ from the original. This is an isotropic distribution.

The $\alpha$-stable distribution is controlled by four parameters: stability index $\alpha$ ( $\alpha \in\langle 0<\alpha \leq 2\rangle$ ), skewness parameter $\beta$, scale parameter $\sigma$ and location parameter $\mu$. The Chambers-Mallows-Stuck method of generation of the $\alpha$-stable symmetric random variables [10] can be used. The method for $\sigma=1$ and $\mu=0$ with a correction for the case where $\alpha=1$ given by Weron in 1996 [1] is presented in (4). To calculate the $\alpha$-stable distributed random variate $X$ two another independent random variates are needed: a random variate $U$, which is uniformly distributed on $[-\pi / 2, \pi / 2]$ and an exponential random variate $W$ obtained with rate parameter $\lambda=1$ :

$$
X=\left\{\begin{array}{l}
S_{\alpha, \beta} \cdot \frac{\sin \alpha\left(U+B_{\alpha, \beta}\right)}{(\cos U)^{1 / \alpha}} \cdot\left(\frac{\cos \left(U-\alpha\left(U+B_{\alpha, \beta}\right)\right)}{W}\right)^{(1-\alpha) / \alpha} \\
\quad \operatorname{iff} \alpha \neq 1 \\
\frac{2}{\pi}\left(\left(\frac{\pi}{2}+\beta U\right) \tan U-\beta \ln \frac{W \cos U}{\frac{\pi}{2}+\beta U}\right), \\
\quad \operatorname{iff} \alpha=1 .
\end{array}\right.
$$

where $B_{\alpha, \beta}=\alpha^{-1} \arctan \left(\beta \tan \frac{\pi \alpha}{2}\right)$, and $S_{\alpha, \beta}=\left(1+\beta^{2} \tan ^{2} \frac{\pi \alpha}{2} 1 /(2 \alpha)\right)$.

In the symmetric version of this distribution (called $S \alpha S$, i.e. symmetric $\alpha$ stable distribution) $\beta$ is set to 0 . For $\alpha=2$ the $S \alpha S(\mu, \sigma)$ distribution reduces to the Gaussian $N(\mu, \sigma)$ and in the case of $\alpha=1$ the Cauchy $C(\mu, \sigma)$ is obtained. In (3) $r_{S \alpha S}$ is a scale parameter.

The difference between the static and the adaptive version of the distribution is in the way of calculation of the distance $d$. In the static version the distance depends on merely the $\alpha$-stable generator. In the adaptive version it depends on the value returned by the generator and multiplied by normalized fitness of the particle. The latter version was inspired by a mutation operator introduced in 12 where it was a component of the immune optimization algorithm called opt-aiNet and designed for multimodal function optimization. The 
mutation operator uses independent random variates for modification of each of the coordinates. This approach gives isotropic distribution for Gaussian random variables but unfortunately turns into non-isotropic for any other $\alpha$-stable distribution in multidimensional search space. We wanted to keep to isotropic distributions, therefore the operator was not migrated as-is. In our adaptive approach the direction is evaluated in the same way like in the static one but the distance is calculated respectively to the current fitness values of the remaining antibodies in $P$ :

$$
d=S \alpha S(0, \sigma) \cdot \exp \left(-f^{\prime}\left(\mathbf{x}_{i}\right)\right)
$$

where $\sigma$ is calculated as in (3) and $f^{\prime}\left(\mathbf{x}_{i}\right)$ is the fitness of the $i$-th solution $\mathbf{x}_{i}$ normalized in $[0,1]$ respectively to the fitness values of all the solutions in $P$ :

$$
\begin{aligned}
f^{\prime}\left(\mathbf{x}_{i}\right) & =\frac{f\left(\mathbf{x}_{i}\right)-f_{\min }}{\left(f_{\max }-f_{\min }\right)}, \\
f_{\max } & =\max _{j=1, \ldots, N} f\left(\mathbf{x}_{j}\right) \text { and } f_{\min }=\min _{j=1, \ldots, N} f\left(\mathbf{x}_{j}\right) .
\end{aligned}
$$
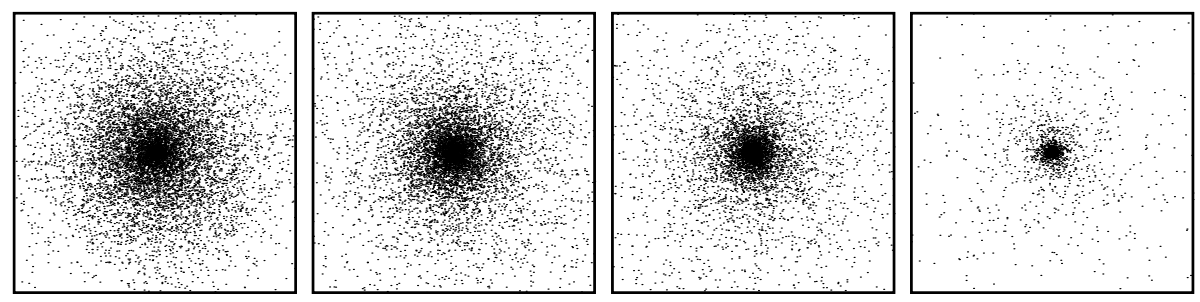

Fig. 1. Distribution of the new points in 2-dimensional search space for $\alpha: 2,1,0.5$, and 0.1

Fig. 1 presents sample distributions of a set of points in the 2-dimensional search space generated for the same original with the static method of generation. There are four distributions for four different values of $\alpha: 2,1,0.5$, and 0.1 .

It often happens that the domain of possible solutions is limited by a set of some box constraints and only the solutions fitted in the constraints are classified as feasible. Both types of distribution presented above allow to generate new locations over the entire domain, so it is possible to generate feasible and unfeasible locations as well. From the theoretical point of view we can easily cope with unfeasible locations simply by allowing them just to stay where they are because the evaluation function formula is usually defined for all points in $\mathbf{R}^{n}$. However, from the engineering point of view we cannot accept such a free treatment since in the real world the constraints are based on the knowledge of the modeled phenomenon and represent its features like e.g. temperature (which cannot be less than $-273 \mathrm{C}$ or higher than some reasonable limit: $+100 \mathrm{C}$ for a water or a smoke point for an oil). Therefore in the presented research it is assumed that the domain of possible solutions is limited by a set of some box constraints and only the solutions fitted in the constraints are classified as feasible. 
Since the main focus in this paper is not about the constrained optimization, we selected a very simple procedure of immediate repairing unfeasible particles. Clearly the $j$-th coordinate of the solution $\mathbf{x}$ breaking its box constraints is trimmed to the exceeded limit, i.e.:

$$
\begin{aligned}
& \text { if } x_{j}<l o_{j} \text { then } x_{j}=l o_{j}, \\
& \text { if } x_{j}>h i_{j} \text { then } x_{j}=h i_{j} .
\end{aligned}
$$

The procedure is applied in the same way to both types of the particles, the classic and the quantum ones. In case of classic particles the velocity vector $\mathbf{v}$ of the repaired particle stays unchanged even if it still leads the particle outside the acceptable search space.

\section{Settings of the Algorithm's Parameters}

The algorithm parameters' settings applied to the experiments presented below originate from [3]. In the cited publication authors present results of experiments obtained for different configurations of swarms tested with the MPB benchmark where there are 10 moving peaks. Among many tested configurations the best results for the optimization problem with 10 moving peaks are obtained where there are 10 sub-swarms and each of them consists of five classic particles and five quantum ones (see Table III in [3]). The total population of particles consists of 100 solutions divided equally into 10 sub-swarms. The values of pure PSO parameters are: $c_{1,2}=2.05$ and $\chi=0.7298$. For QSO the range of exclusion is set to 31.5 (for the best performance the value of $\rho$ should be set close to 30. However, the precision of this parameter's setting is not crucial. In [3] the authors claim that the algorithm is not very sensitive to small changes of $\rho$ ).

In the presented algorithm there is no strategy of detecting the appearance of change in the fitness landscape. Since our main goal was studying the properties of the different distributions of the quantum particles, we assumed that it would just introduce yet another unnecessary bias into the obtained values of offline error and make their analysis even more difficult. Therefore a change is known to the system instantly as it appears and there is not any additional computational effort for its detection. When the change appears, all the solutions stored in both classic and quantum particles are reevaluated and the swarm memory is forgotten. Classic particle's attractors are overwritten by current solutions represented by these particles and sub-swarms' attractors are overwritten by the current best solutions in the sub-swarms.

\section{Applied Measure and the Benchmark}

In the performed experiments the offline error (briefly oe) measure [7/13] of obtained results was used. The offline error represents the average deviation from the optimum of the fitness of the best individual evaluated since the last change of the fitness landscape. Every time the solution's fitness is evaluated, 
an auxiliary variable is increased by the value which is the deviation of the best solution evaluated since the last change including the one just evaluated as well. When the experiment is finished the sum in the variable is divided by the total number of evaluations and returned as the offline error. Formally:

$$
o e=\frac{1}{N_{c}} \sum_{j=1}^{N_{c}}\left(\frac{1}{N_{e}(j)} \sum_{i=1}^{N_{e}(j)}\left(f_{j}^{*}-f_{j i}^{*}\right)\right),
$$

where $N_{c}$ is the total number of changes of the fitness landscape in the experiment, $N_{e}(j)$ is the number of evaluations of the solutions performed for the $j$-th state of the landscape, $f_{j}^{*}$ is the value of optimal solution for the $j$-th landscape (i.e. between the $j$-th and $(j+1)$-th change in the landscape) and $f_{j i}^{*}$ is the current best found fitness value for the $j$-th landscape i.e. the best value found among the ones belonging to the set from $f_{j 1}$ till $f_{j i}$ where $f_{j i}$ is the value of the fitness function returned for its $i$-th call performed for the $j$-th landscape.

During the process of search the offline error can be calculated in two ways: in one of them the error is evaluated from the beginning of the experiment while in another one - the value of offline error starts to be evaluated only after some number of changes in the fitness landscape. The latter way is advised as saddled with the less measurement error caused by the initial phase of the search process (please see e.g. 14] for a discussion about the possible influence of the initial phase on the quality of the results obtained for MPB). Therefore, just this way was applied in our tests.

For compatibility with experiments published by others the number of evaluations between subsequent changes of the fitness landscape equals 5000. During a single experiment the fitness landscape changes 110 times (however for the first 10 changes the error is not evaluated). Every experiment was repeated 50 times and the means are presented.

The parameters of MPB were set exactly the same as specified in 3 in scenario 2. The fitness landscape was defined for the 5-dimensional search space with boundaries for each of dimensions set to $\langle 0 ; 100\rangle$. For the search space there exist a set of 10 moving peaks which vary their height randomly within the interval $\langle 30 ; 70\rangle$, width within $\langle 1 ; 12\rangle$ and position by a distance of 1 .

\section{$6 \quad$ Results of Experiments}

We started our research from repeating some of the experiments presented in [3]. It was necessary to make the earlier results comparable to the current ones since in our case a period of the first 10 changes in the environment is excluded from calculating the offline error which makes the values of oe significantly smaller than those in 3. We repeated experiments with uniform distribution of new locations inside a quantum cloud (called further $M_{\text {cloud }}$ ) for a series of values of $r_{\text {cloud }}$ : from 0.05 to 4.5 with step 0.05 . The three best values of oe were: 1.6264 (std.dev.: 0.4104), 1.6297 (std.dev.: 0.4062), 1.6298 (std.dev.: 0.5227) and they were obtained for $r_{\text {cloud }}: 0.30,0.35$ and 0.25 respectively. 

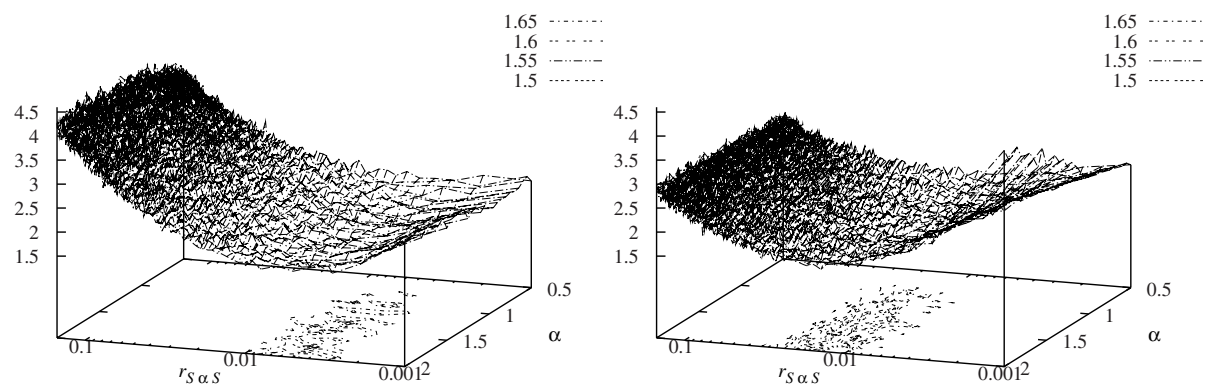

Fig. 2. Offline error for $M_{\alpha s t a t i c}$ i.e. a static version (on the left) and for $M_{\text {aadapt }}$ i.e. an adaptive version (on the right): $r_{S \alpha S}$ vs. $\alpha$

Table 1. The three best values of offline error obtained for the two tested methods of the new location generation for the case with 10 moving peaks

\begin{tabular}{|c|c|c|c|c|}
\hline method & offline error & std. dev. & $\sigma$ & $\alpha$ \\
\hline$M_{\alpha s t a t i c}[1]$ & 1.4603 & 0.3066 & 0.25 & 1.35 \\
\hline$M_{\alpha s t a t i c}[2]$ & 1.4665 & 0.4188 & 0.25 & 1.80 \\
\hline$M_{\text {ostatic }}[3]$ & 1.5023 & 0.3518 & 0.35 & 1.00 \\
\hline$M_{\text {oadapt }}[1]$ & 1.4614 & 0.3255 & 0.60 & 1.70 \\
\hline$M_{\text {oadapt }}[2]$ & 1.4722 & 0.3041 & 0.85 & 1.70 \\
\hline$M_{\text {oadapt }}[3]$ & 1.5008 & 0.3496 & 0.60 & 1.75 \\
\hline
\end{tabular}

In our research we wanted to get performance characteristics of the tested distributions first. This way we are able to compare not only the best results possible to obtain for a given test-case but also an information about the robustness of the searching engines and its sensitivity to changes in their parameters settings. Thus, two large groups of experiments were performed. We tested the static version of $\alpha$-stable symmetric distribution $-M_{\alpha s t a t i c}$, and adaptive version of $\alpha$-stable symmetric distribution $-M_{\alpha a d a p t}$. The sets of tests with the two methods were based on variation of values of two method's parameters: $\alpha$ and $r_{S \alpha S}$. The former parameter varied from 0.5 to 2 with step 0.05 while the latter - from 0.001 to 0.1 with step 0.001. It gave 3100 configurations for each of the approaches. They were tested on the same class of dynamic environments build by MPB benchmark with 10 moving peaks and with its parameters set to values as defined above. Obtained values of offline error for the two groups of experiments are presented in Fig. 2.

In Table 1 for each of the distributions the best three configurations and the values of offline error for each of the three are presented. The offline error for $M_{\text {cloud }}$ is higher than for the two remaining methods. The significance levels obtained with Student's t tests indicate difference between $M_{\text {cloud }}$ and distributions in unlimited area i.e. for both tests between $M_{\text {cloud }}$ and the two methods the 

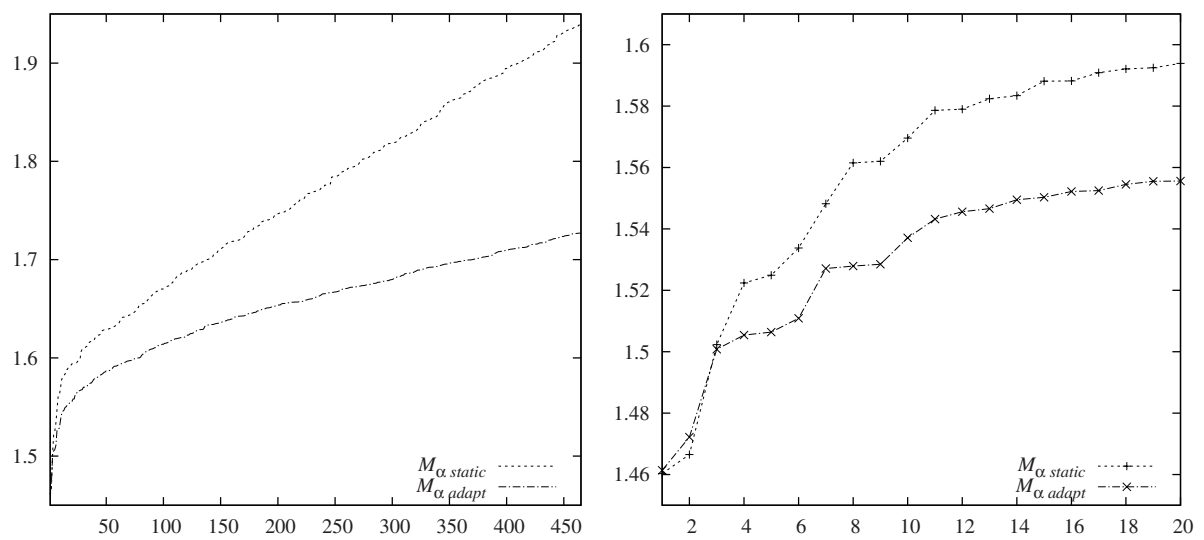

Fig. 3. The first $10 \%$ of values of offline error sorted ascending for the two tested distributions - left side graph, and a zoom to the best first 20 values - right side graph

significance level is lower than the commonly accepted level of 0.05 (for $M_{\alpha s t a t i c}$ $-p=0.025437$, for $\left.M_{\alpha a d a p t}-p=0.029881\right)$.

Apart from Fig. 2 and Table 1 yet another quantitative comparison was performed based on the same set of results. The analysis is presented in Fig. 3 To generate Fig. 3 for each of the methods, the values of offline error obtained for tested configurations of parameters were sorted ascending. This way we can observe sensitivity of the methods for changes in the parameters values as well as the small disturbances in the optimized fitness landscape. We can compare not only the best results but also the number of other configurations giving satisfying results i.e. the size of area of useful configurations of the distributions. A graph with the first $10 \%$ of sorted values of offline error is in Fig. 3 .

Both curves in Fig. 3 start from the level of offline error which is presented in Table 1 and which is almost the same for each of them. However they quickly branch. It is clearly visible that the adaptive method outperforms the static method. The advantage is that the adaptive method is much more tolerant for lack of fitting the methods parameters to the properties of the fitness landscape. In other words an appropriately tuned algorithm is doing very well for each of the methods however if the algorithm is not perfectly tuned to the problem, the loss of performance is much less for adaptive methods of distribution.

\section{Conclusions}

In this paper two methods of evaluation of the quantum particle's new position are experimentally verified: the static and the adaptive one. Both methods employ the $\alpha$-stable symmetrically distributed random variable. The distribution is controlled by the parameter $\alpha$, which is responsible for the density of distribution of new locations around the quantum particle. Obtained results are satisfactory: they are better than those for uniform distribution of new locations 
in the limited area around the quantum particle. Besides the results of series of experiments visualized in Fig. 3] showed that the adaptive method of distribution is less sensitive to small changes in its parameters than the static one.

\section{References}

1. Blackwell, T.: Particle Swarm Optimization in Dynamic Environments. In: Evolutionary Computation in Dynamic and Uncertain Environments. Studies in Computational Intelligence, vol. 51, pp. 29-49. Springer, Heidelberg (2007)

2. Blackwell, T., Branke, J.: Multi-swarm optimization in dynamic environments. In: Raidl, G.R., Cagnoni, S., Branke, J., Corne, D.W., Drechsler, R., Jin, Y., Johnson, C.G., Machado, P., Marchiori, E., Rothlauf, F., Smith, G.D., Squillero, G. (eds.) EvoWorkshops 2004. LNCS, vol. 3005, pp. 489-500. Springer, Heidelberg (2004)

3. Blackwell, T., Branke, J.: Multiswarms, exclusion, and anti-convergence in dynamic environments. IEEE Trans. Evol. Comput. 10(4), 459-472 (2006)

4. Li, X.: Adaptively choosing neighborhood bests in a particle swarm optimizer for multimodal function optimization. In: Deb, K., et al. (eds.) GECCO 2004. LNCS, vol. 3102, pp. 105-116. Springer, Heidelberg (2004)

5. Li, X., Branke, J., Blackwell, T.: Particle swarm with speciation and adaptation in a dynamic environment. In: GECCO 2006: Proc. Conf. on Genetic and Evolutionary Computation, pp. 51-58. ACM Press, New York (2006)

6. Parrot, D., Li, X.: Locating and tracking multiple dynamic optima by a particle swarm model using speciation. IEEE Trans. Evol. Comput. 10(4), 440-458 (2006)

7. Branke, J.: Memory enhanced evolutionary algorithm for changing optimization problems. In: Proc. of the Congress on Evolutionary Computation, pp. 1875-1882. IEEE Press, Piscataway (1999)

8. Clerc, M., Kennedy, J.: The particle swarm-explosion, stability, and convergence in a multi-dimensional complex space. IEEE Trans. Evol. Comput. 6(1), 58-73 (2002)

9. Marsaglia, G.: Choosing a point from the surface of a sphere. Ann. Math. Statist. 43(2), 645-646 (1972)

10. Chambers, J.M., Mallows, C.L., Stuck, B.W.: A method for simulating stable random variables. J. Amer. Statist. Assoc. 71(354), 340-344 (1976)

11. Weron, R.: On the Chambers-Mallows-Stuck method for simulating skewed stable random variables. Statist. Probab. Lett. 28, 165-171 (1996)

12. de Castro, L.N., Timmis, J.: An artificial immune network for multimodal function optimization. In: Proc. of the IEEE Congress on Evolutionary Computation, vol. 1, pp. 674-699. IEEE Press, Piscataway (2002)

13. Branke, J.: Evolutionary Optimization in Dynamic Environments. Kluwer Academic Publishers, Dordrecht (2002)

14. Trojanowski, K.: B-cell algorithm as a parallel approach to optimization of moving peaks benchmark tasks. In: Sixth International Conf. on Computer Information Systems and Industrial Management Applications (CISIM 2007), IEEE Computer Society Conf. Publishing Services, pp. 143-148 (2007) 